\title{
Open Bisimulations on Chi Processes ${ }^{\star}$
}

\author{
Yuxi $\mathrm{Fu}^{\star \star}$ \\ Department of Computer Science \\ Shanghai Jiaotong University, Shanghai 200030, China
}

\begin{abstract}
The paper carries out a systematic investigation into the axiomatization problem of the asymmetric chi calculus. As a crucial step in attacking the problem, an open style bisimilarity is defined for each of the eighteen L-bisimilarities and the two are proved to be equal. On top of the open bisimilarities, explicit definitions of the eighteen L-congruences are given, which suggest immediately possible axioms for the congruence relations. In addition to the axioms for strong bisimilarity, the paper proposes altogether twenty one additional axioms, three of which being the well-known tau laws and the other eighteen being new. These axioms help to lift a complete system for the strong bisimilarity to complete systems for the eighteen L-congruences.
\end{abstract}

\section{Introduction}

The $\chi$-calculus $([1,2,5])$ is a recent addition to the family of calculi of mobile process ([8]). It is a process algebraic formalization of reaction graph ([4]). The latter is proposed to emphasize the graphical aspect of concurrent computational objects. The language is a further step towards a more abstract model of concurrent computation. One of its novel features is a uniform treatment of names. Uniformity supports the idea that there should be no difference between input and output prefixes. The followings are examples of communication in $\chi$ :

$$
\begin{gathered}
(x)(a[x] . P|\bar{a}[y] \cdot Q| R) \stackrel{\tau}{\longrightarrow} P[y / x]|Q[y / x]| R[y / x] \\
(x)(\bar{a}[x] . P|a[y] \cdot Q| R) \stackrel{\tau}{\longrightarrow} P[y / x]|Q[y / x]| R[y / x] \\
(x) a[x] \cdot P|\bar{a}[y] \cdot Q \stackrel{\tau}{\longrightarrow} P[y / x]| Q \\
(x) \bar{a}[x] \cdot P|a[y] \cdot Q \stackrel{\tau}{\longrightarrow} P[y / x]| Q
\end{gathered}
$$

Here $a[x] . P$ and $\bar{a}[y] . Q$ are processes in prefix form, in which $x$ and $y$ are global. In $(x)(a[x] \cdot P|\bar{a}[y] \cdot Q| R)$ the name $x$ is local as it is restricted by a localization operator $(x)$. In (1) and (2) the interactions between $a[x] . P$ and $\bar{a}[y] . Q$ cause the local name $x$ to be replaced by $y$ throughout the term over which the localization operator $(x)$ applies. In (3) and (4) the interactions do not affect $Q$ as it is

^ CONCUR '99, Lecture Notes in Computer Science 1664, 304-319, 1999.

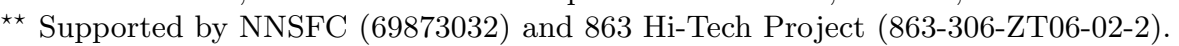


not restricted by $(x)$. The four reductions should demonstrate the symmetry of communications in $\chi$-calculus.

If one insists that there should be a difference between positive prefix operation $a[x]$ and negative prefix operation $\bar{a}[x]$ then one obtains an asymmetric version of $\chi$-calculus. In asymmetric $\chi$-calculus, reductions (1) and (3) are admissible whereas reductions (2) and (4) are illegal. Asymmetric and polyadic versions of $\chi$-calculus have been studied by Parrow and Victor in [10,11].

The equational theory of mobile processes has attracted a lot of attention. Lin has axiomatized successfully some weak congruences on mobile processes ([7]). He concluded that Milner's three tau laws are enough to lift system for strong congruences to system for weak congruences in calculus of mobile processes. So far all complete systems for weak congruences on mobile processes are essentially of symbolic nature. An alternative is used by Sangiorgi in his study of open bisimulation ([12]). Compared to symbolic approach, the open approach has the virtue of simplicity. Strong open bisimilarity on finite mobile processes can be easily axiomatized. Axiomatization of weak open congruence however has not been seriously considered.

In this paper we answer some of the open problems in the theory of mobile processes. Our main contributions are as follows:

- The paper improves our understanding of the asymmetric $\chi$-calculus by studying open bisimilarities. For each of the eighteen distinct $L$-bisimilarities, we introduce an open bisimilarity that coincides with the $L$-bisimilarity.

- Axiomatization for $L$-congruences on asymmetric $\chi$-processes has not been considered before. We give in this paper complete systems for all the eighteen distinct $L$-congruences. Our result brings out the importance of the open counterparts of $L$-congruences.

- In $[11,3]$ attempts have been made to give complete systems for weak congruence on polyadic $\chi$-processes, and respectively, four $L$-congruences on symmetric $\chi$-processes. In this paper it is pointed out that all the proofs establishing the claimed completeness are wrong. A way to correct the mistake is proposed.

- As a byproduct, the paper provides a complete system for barbed congruence ([9]) on asymmetric $\chi$-processes. It is demonstrated that bisimulation lattice is of great help in obtaining such a system.

- Axiomatization for weak open congruence on $\pi$-processes has not been paid enough attention. The approach used in this paper can be applied to give immediately a complete system for weak open congruence on $\pi$-processes.

- The paper refutes the general belief that Milner's three tau-laws are sufficient, in calculi of mobile processes, to lift a complete system for a strong congruence to a complete system for the corresponding weak congruence. This is related to the failure of Hennessy Lemma in such calculi.

Due to space restriction, most of the proofs are omitted in this extended abstract. The proofs given are sketchy. Some of the intermediate lemmas are excluded. A much more detailed account can be found in the full paper [6]. In the rest of the paper we will leave out the adjective in "asymmetric $\chi$ ". 


\section{Background}

Let $\mathcal{N}$ be the set of names ranged over by small case letters and $\overline{\mathcal{N}}$ the set $\{\bar{x} \mid x \in \mathcal{N}\}$ of conames. The Greek letter $\alpha$ ranges over $\mathcal{N} \cup \overline{\mathcal{N}}$. For $\alpha \in \mathcal{N} \cup \overline{\mathcal{N}}$, $\bar{\alpha}$ is defined as $a$ if $\alpha=\bar{a}$ and as $\bar{a}$ if $\alpha=a$. The $\chi$-processes are defined by the following abstract grammar: $P:=\mathbf{0}|\alpha[x] . P| P|P|(x) P|[x=y] P| P+P$. Most of the combinators have completely the same reading as those of the $\pi$ calculus. The name $x$ in $(x) P$ is local. A name is global in $P$ if it is not local in $P$. For instance the name $x$ in both $a[x] . P$ and $\bar{a}[x] . P$ is global. We will write $g n(P)$ for the set of global names in $P$. As this paper is mainly concerned with axiomatization of finite $\chi$-processes, we have omitted the replication operator. The set of $\chi$-processes will be denoted by $\mathcal{C}$. The well-known $\alpha$-convention will be adopted.

Let $\delta$ range over the set $\{\tau\} \cup\{m[x], \bar{m}[x], m x, \bar{m}(x),[y / x],(y / x] \mid m, x, y \in$ $\mathcal{N}\}$ of transition labels and $\mu$ over $\{\tau\} \cup\{m[x], \bar{m}[x], m x, \bar{m}(x) \mid m, x \in \mathcal{N}\}$. In $(y / x], x$ and $y$ must be different. A name in $\delta$ is local if it appears as $x$ in $\bar{m}(x)$ or $(x / y]$; it is global otherwise. Let $\ln (\delta)$, respectively $g n(\delta)$, denote the set of local, respectively global, names appearing in $\delta$; and let $n(\delta)$ denote the set of names in $\delta$. The sets $\ln (\mu), g n(\mu)$ and $n(\mu)$ are defined accordingly.

The following rules define the operational semantics of $\chi$-calculus:

$$
\begin{aligned}
& \underset{\alpha[x] . P \stackrel{\alpha[x]}{\longrightarrow} P}{\frac{P \stackrel{\delta}{\longrightarrow} P^{\prime}}{[x=x] . P \stackrel{\delta}{\longrightarrow} P^{\prime}}} \quad \frac{P \stackrel{\delta}{\longrightarrow} P^{\prime}}{P+Q \stackrel{\delta}{\longrightarrow} P^{\prime}} \\
& \frac{P \stackrel{\mu}{\longrightarrow} P^{\prime} \quad \ln (\mu) \cap \operatorname{gn}(Q)=\emptyset}{P\left|Q \stackrel{\mu}{\longrightarrow} P^{\prime}\right| Q} \\
& \frac{P \stackrel{[y / x]}{\longrightarrow} P^{\prime}}{P\left|Q \stackrel{[y / x]}{\longrightarrow} P^{\prime}\right| Q[y / x]} \quad \frac{P \stackrel{(y / x]}{\longrightarrow} P^{\prime} y \notin g n(Q)}{P\left|Q \stackrel{(y / x]}{\longrightarrow} P^{\prime}\right| Q[y / x]} \\
& \frac{P \stackrel{m x}{\longrightarrow} P^{\prime} Q \stackrel{\bar{m}[x]}{\longrightarrow} Q^{\prime}}{P\left|Q \stackrel{\tau}{\longrightarrow} P^{\prime}\right| Q^{\prime}} \quad \frac{P \stackrel{m x}{\longrightarrow} P^{\prime} \quad Q \stackrel{\bar{m}(x)}{\longrightarrow} Q^{\prime} \quad x \notin g n(P)}{P \mid Q \stackrel{\tau}{\longrightarrow}(x)\left(P^{\prime} \mid Q^{\prime}\right)} \\
& \frac{P \stackrel{m[x]}{\longrightarrow} P^{\prime}}{P\left|Q \stackrel{[y / x]}{\longrightarrow} P^{\prime}[y / x]\right| Q^{\prime}[y / x]} Q^{\prime} \quad \frac{P \stackrel{m[x]}{\longrightarrow} P^{\prime} \quad Q \stackrel{\bar{m}(y)}{\longrightarrow} Q^{\prime} \quad y \notin g n(P)}{P\left|Q \stackrel{(y / x]}{\longrightarrow} P^{\prime}[y / x]\right| Q^{\prime}[y / x]} \\
& \frac{P \stackrel{\delta}{\longrightarrow} P^{\prime} x \notin n(\delta)}{(x) P \stackrel{\delta}{\longrightarrow}(x) P^{\prime}} \quad \frac{P \stackrel{m[x]}{\longrightarrow} P^{\prime} \quad x \neq m}{(x) P \stackrel{m y}{\longrightarrow} P^{\prime}[y / x]} \quad \frac{P \stackrel{\bar{m}[x]}{\longrightarrow} P^{\prime} x \neq m}{(x) P \stackrel{m(x)}{\longrightarrow} P^{\prime}} \\
& \frac{P \stackrel{[y / x]}{\longrightarrow} P^{\prime} \quad x \neq y}{(x) P \stackrel{\tau}{\longrightarrow} P^{\prime}} \quad \frac{P \stackrel{[y / x]}{\longrightarrow} P^{\prime} x \neq y}{(y) P \stackrel{(y / x]}{\longrightarrow} P^{\prime}} \quad \frac{P \stackrel{(y / x]}{\longrightarrow} P^{\prime}}{(x) P \stackrel{\tau}{\longrightarrow}(y) P^{\prime}} \quad \frac{P \stackrel{[x / x]}{\longrightarrow} P^{\prime}}{(x) P \stackrel{\tau}{\longrightarrow}(x) P^{\prime}}
\end{aligned}
$$

The semantics is different from the one in $[10,3]$. Here $\bar{m}[x] \cdot P|m[x] . Q \stackrel{[x / x]}{\longrightarrow} P| Q$ but not $\bar{m}[x] . P|m[x] . Q \stackrel{\tau}{\longrightarrow} P| Q$. In $[10,3],[x / x]$ is identified with $\tau$; here they are different. 
Some notations need be fixed before we proceed to next section. Let $\Longrightarrow$ be the reflexive and transitive closure of $\stackrel{\tau}{\longrightarrow}$. We will write $\stackrel{\delta}{\Longrightarrow}$ for $\Longrightarrow \stackrel{\delta}{\Longrightarrow} \Longrightarrow$. We will also write $\stackrel{\widehat{\delta}}{\Longrightarrow}$ for $\stackrel{\delta}{\Longrightarrow}$ if $\delta \neq \tau$ and for $\Longrightarrow$ otherwise. The notation $\Longrightarrow_{x}$ will stand for $\Longrightarrow \stackrel{[x / x]}{\longrightarrow} \Longrightarrow \stackrel{[x / x]}{\longrightarrow} \ldots \Longrightarrow \stackrel{[x / x]}{\longrightarrow} \Longrightarrow$, where the transition $\stackrel{[x / x]}{\longrightarrow}$ occurs zero or a finite number of times. It follows from definition that $\Longrightarrow \subseteq \Longrightarrow_{x}$. For simplification $\stackrel{\delta}{\Longrightarrow} \Longrightarrow x$ will be abbreviated to $\stackrel{\delta}{\Longrightarrow}$. An atomic substitution of $y$ for $x$ is denoted by $[y / x]$. A general substitution $\sigma$ is the composition of atomic substitutions, whose effect on a process $P$ is defined by $P\left[y_{1} / x_{1}\right] \ldots\left[y_{n} / x_{n}\right] \stackrel{\text { def }}{=}$ $\left(P\left[y_{1} / x_{1}\right] \ldots\left[y_{n-1} / x_{n-1}\right]\right)\left[y_{n} / x_{n}\right]$. The composition of zero atomic substitution is an empty substitution [] whose effect on a process is vacuous. A sequence of names $x_{1}, \ldots, x_{n}$ will be abbreviated as $\boldsymbol{x}$; and consequently $\left(x_{1}\right) \ldots\left(x_{n}\right) P$ will be abbreviated to $(\boldsymbol{x}) P$. When the length of $\boldsymbol{x}$ is zero, $(\boldsymbol{x}) P$ is just $P$.

In the rest of the paper $M$ and $N$, and their indexed forms, denote finite lists of equalities $x=y$. Let $M$ be $x_{1}=y_{1}, \ldots, x_{n}=y_{n}$. Then $[M] P$ denotes $\left[x_{1}=y_{1}\right] \ldots\left[x_{n}=y_{n}\right] P$. If $M$ logically implies $N$, we write $M \Rightarrow N$; and if both $M \Rightarrow N$ and $N \Rightarrow M$ we write $M \Leftrightarrow N$. If $M$ is an empty list, it plays the role of logical truth, in which case $[M] P$ is just $P$. Clearly a list $M$ of match equalities defines an equivalence relation on the set $n(M)$ of names appearing in $M$. We use $\sigma_{M}$ to denote an arbitrary substitution that sends all members of an equivalence class to a representative of that class and sends a name not in $n(M)$ to itself. For a finite number of processes $P_{i}, i \in I$, we write $\sum_{i \in I} P_{i}$ for $P_{1}+\ldots+P_{n}$. We have leave out the parentheses in $P_{1}+\ldots+P_{n}$ as + is associative both semantically and proof theoretically.

In order to axiomatize the congruence relations of this paper, we need to internalize, as it were, the labels of the transition system. In the following definition $a$ is fresh: $\alpha(x) . P \stackrel{\text { def }}{=}(x) \alpha[x] . P$, where $x \notin\{\alpha, \bar{\alpha}\} ; \tau . P \stackrel{\text { def }}{=}(a)[a / a] . P$; $[y / x] . P \stackrel{\text { def }}{=}(a)(\bar{a}[y] \mid a[x] . P) ;(y / x] . P \stackrel{\text { def }}{=}(y)[y / x] . P$, where $x \neq y$. The prefix $[y / x]$, first introduced in $[1,10]$, is called an update. It is clear from definition that both $x$ and $y$ in $[y / x] . P$ are global. On the other hand the $y$ in the restricted update $(y / x] . P$ is local.

We state below some technical results to be used in the rest of the paper. The proofs of which are simple inductions on derivation.

Lemma 1. Let $n(\sigma)$ denote the names appearing in the substitution $\sigma$.

(i) If $n(\sigma) \cap \ln (\mu)=\emptyset$ and $P \stackrel{\mu}{\longrightarrow} P^{\prime}$ then $P \sigma \stackrel{\mu \sigma}{\longrightarrow} P^{\prime} \sigma$.

(ii) If $P \stackrel{[y / x]}{\longrightarrow} P^{\prime}$ then $P \sigma \stackrel{[y \sigma / x \sigma]}{\longrightarrow} P^{\prime} \sigma[y \sigma / x \sigma]$.

(iii) If $y \notin n(\sigma)$ and $P \stackrel{(y / x]}{\longrightarrow} P^{\prime}$ then $P \sigma \stackrel{(y / x \sigma]}{\longrightarrow} P^{\prime} \sigma[y / x \sigma]$.

Lemma 2. If $(x) P \Longrightarrow(y) P^{\prime}$, then either $P \Longrightarrow_{x} P^{\prime}$ and $x=y$, or $P \Longrightarrow_{x} \stackrel{(y / x]}{\Longrightarrow}$ $P^{\prime}$, or, for $y_{1}, \ldots, y_{n}, n \geq 1, P \Longrightarrow x \stackrel{\left(y_{1} / x\right]}{\Longrightarrow} y_{1}{\stackrel{\left(y_{2} / y_{1}\right]}{\longrightarrow}}_{y_{2}} \ldots \stackrel{\left(y_{n} / y_{n-1}\right]}{\Longrightarrow} y_{n} \stackrel{\left(y / y_{n}\right]}{\Longrightarrow} P_{y}^{\prime}$.

We refer the reader to $[2,3]$ for more on the semantics of $\chi$-calculus. 


\section{Bisimulation Lattice}

Bisimulation equalities are the finest equivalence relation on processes. For a particular process calculus, there is not just one weak bisimulation equality but a whole range of them. These equalities differ in the extent actions are admitted. In practice one uses one bisimulation equality in preference to others because the processes one is interested in are capable of performing only certain kinds of actions.

We will define a class of bisimulation equalities on $\chi$-processes induced by different sets of admissible actions. For that purpose, we introduce the following notations. Let $f o$ denote the set $\{\bar{a}[x] \mid a, x \in \mathcal{N}\}$ of free outputs, $f i$ the set $\{a[x] \mid x \in \mathcal{N}\}$ of free inputs, $i$ the set $\{a x \mid x \in \mathcal{N}\}$ of inputs, ro the set $\{\bar{a}(x) \mid a, x \in \mathcal{N}\}$ of restricted outputs, $u$ the set $\{[y / x] \mid x, y \in \mathcal{N}\}$ of updates and $r u$ the set $\{(y / x] \mid x, y \in \mathcal{N}\}$ of restricted updates. Define $\mathcal{L}$ as $\{\cup S \mid S \subseteq$ $\{f o, f i, i, r o, u, r u\} \wedge S \neq \emptyset\}$.

Contexts are certain processes with a hole. They are inductively defined as follows: (i) [] is a context; (ii) if $C[]$ is a context then $C[]|P, P| C[],(x) C[]$ and $\alpha[x] . C[]$ are contexts. A binary relation $\mathcal{R}$ on $\mathcal{C}$ is closed under context if $P \mathcal{R} Q$ implies $C[P] \mathcal{R} C[Q]$ for every context $C[]$. It is closed under substitution if $P \mathcal{R} Q$ implies $P \sigma \mathcal{R} Q \sigma$ for every substitution $\sigma$.

Definition 3. Let $\mathcal{R}$ be a binary symmetric relation on $\mathcal{C}$ and $L$ be an element of $\mathcal{L}$. The relation $\mathcal{R}$ is an L-relation if whenever $P \mathcal{R} Q$ and $P \stackrel{\phi}{\longrightarrow} P^{\prime}$, for $\phi \in L \cup\{\tau\}$, then some $Q^{\prime}$ exists such that $Q \stackrel{\widehat{\phi}}{\Longrightarrow} Q^{\prime} \mathcal{R} P^{\prime}$. An open L-relation is an L-relation that is closed under substitution. An L-bisimulation is an $L$ relation that is closed under context. The L-bisimilarity, notation $\approx_{L}$, is the largest L-bisimulation.

According to Definition 3, $P$ is $L$-bisimilar to $Q$ if an admissible action $\phi$ of $P$, that is $\phi \in L$, can be simulated by the same action from $Q$ up to tau actions and vice versa. Closedness under context guarantees that $L$-bisimilarity is stable with respect to all but the summation operation.

Theorem 4. If $\sigma$ is a substitution, $L \in \mathcal{L}, P \approx_{L} Q$ and $O \in \mathcal{C}$ then (i) $\alpha[x] . P \approx_{L} \alpha[x] . Q ;$ (ii) $P\left|O \approx_{L} Q\right| O$; (iii) $(x) P \approx_{L} \quad(x) Q$; (iv) $[x=y] P \approx_{L}$ $[x=y] Q ;(v) P \sigma \approx_{L} Q \sigma$.

There are altogether $63 \mathrm{~L}$-bisimilarities. Not all of them are distinct. The next theorem reveals the full picture of the order relationship among them.

Theorem 5. Suppose $L, L_{1}, L_{2} \in \mathcal{L}$. Then the following properties hold:

(i) $\approx_{L} \subseteq \approx_{i}$ and if $L \neq i$ then the inclusion is strict.

(ii) $\approx_{L_{1}} \nsubseteq \approx_{L_{2}}$ if either $\left(f i \cap L_{1}=\emptyset\right) \wedge\left(f i \subseteq L_{2}\right)$ or $\left(r u \cap L_{1}=\emptyset\right) \wedge\left(r u \subseteq L_{2}\right)$ or $\left((r u \cup r o) \cap L_{1}=\emptyset\right) \wedge\left(r o \subseteq L_{2}\right)$ or $\left(u \cap L_{1}=\emptyset\right) \wedge\left(u \subseteq L_{2}\right)$ or $\left((u \cup f o) \cap L_{1}=\right.$ $\emptyset) \wedge\left(f o \subseteq L_{2}\right)$.

(iii) $\approx_{r u} \subset \approx_{r o}$ and $\approx_{u} \subset \approx_{f o}$. Both inclusions are strict. 


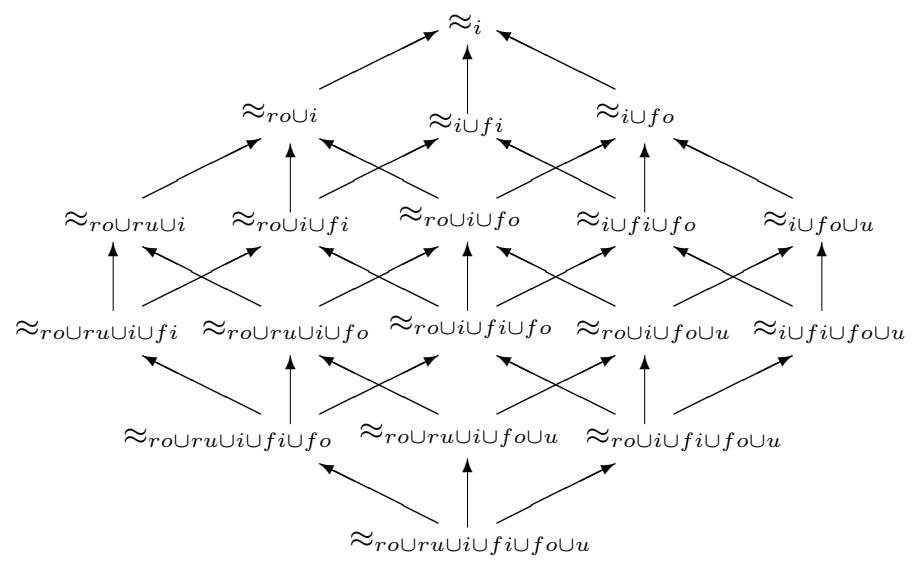

Fig. 1. The Bisimulation Lattice of Chi Calculus

It follows from Theorem 5 that there are altogether 18 distinct $L$-bisimilarities. These are described in Figure 1. In the diagram an arrow indicates a strict inclusion. Each labeled node is the principal representative of a number of $L$ bisimilarities that coincide. For instance, the node labeled by $\approx_{r o \cup r u \cup i \cup f i}$ is the principal representative of the equivalence class $\left\{\approx_{L} \mid r u \cup f i \subseteq L \subseteq\right.$ ro $\cup$ $r u \cup i \cup f i$ and $L \in \mathcal{L}\}$. The order structure induced by the arrow relation is called the bisimulation lattice of $\chi$-calculus. Due to space limitation we will be concentrating on $\approx_{r o \cup r u \cup i \cup f i \cup f o \cup u}$ and $\approx_{r o \cup r u \cup i \cup f i \cup f o}$ in this paper. Extensive studies of the other sixteen can be found in the full paper.

The proof of Theorem 5 is sketched in [3]. In this paper we give some examples to support the inequality claimed in the theorem. These examples are far more general than the ones in [3] in the sense that they are axiom generating.

Suppose $x \neq y$ and $L \cap f i=\emptyset$. Then

$$
\begin{aligned}
& m(y) \cdot(P+[y / x] \cdot Q) \not_{f i} m(y) \cdot(P+[y / x] \cdot Q)+m[x] \cdot Q[x / y] \\
& m(y) \cdot(P+[y / x] \cdot Q) \approx_{L} m(y) \cdot(P+[y / x] \cdot Q)+m[x] \cdot Q[x / y]
\end{aligned}
$$

Now (5) is obvious whereas (6) is slightly more subtle. None of the actions confined in $L$ can tell the two processes apart. The reduction $(m(y) \cdot(P+[y / x] \cdot Q)+$ $m[x] . Q[x / y]) \mid \bar{m}[z] \stackrel{[z / x]}{\longrightarrow} Q[z / y][z / x]$, for instance, is matched up by $m(y) .(P+$ $[y / x] . Q) \mid \bar{m}[z] \stackrel{\tau}{\longrightarrow}[z / x] . Q[z / y] \stackrel{[z / x]}{\longrightarrow} Q[z / y][z / x]$.

Suppose $Q$ cannot perform any restricted updates up to tau actions. Then

$$
\begin{aligned}
(y / x] \cdot Q+\tau \cdot Q[x / y] & \not_{r u} \tau \cdot Q[x / y] \\
(y / x] \cdot(P+(z / y] \cdot Q) & \not_{r u}(y / x] \cdot(P+(z / y] \cdot Q)+(z / x] \cdot Q[z / y] \\
(y / x] \cdot Q+\tau \cdot Q[x / y] & \approx_{L} \tau \cdot Q[x / y] \\
(y / x] \cdot(P+(z / y] \cdot Q) & \approx_{L}(y / x] \cdot(P+(z / y] \cdot Q)+(z / x] \cdot Q[z / y]
\end{aligned}
$$


when $L \cap r u=\emptyset$. Both (7) and (8) are obvious. Intuitively (9) holds because if the admissible actions are confined in $L$ then the first action of $(y / x] . Q$ is not fireable. For it to be activated the global name $x$ must be localized in a context. But then the action invoked by $(y / x]$ amounts to substituting the local $y$ for the local $x$, the side effect being the same as that of applying the $\alpha$-conversion. For similar reason (10) holds because replacing $x$ by the local $z$ in $Q[z / y]$ results in the same process as the one obtained by first substituting the local $y$ for $x$ in $Q$ and then substituting the local $z$ for $y$.

If $Q$ can perform a restricted output action not matched up by $P$ then

$$
\begin{aligned}
& \bar{a}(x) \cdot(P+(y / x] \cdot Q) \neq_{r o} \bar{a}(x) \cdot(P+(y / x] \cdot Q)+\bar{a}(y) \cdot Q[y / x] \\
& \bar{a}(x) \cdot(P+(y / x] \cdot Q) \approx_{L} \bar{a}(x) \cdot(P+(y / x] \cdot Q)+\bar{a}(y) \cdot Q[y / x]
\end{aligned}
$$

when $L \cap(r u \cup r o)=\emptyset$. The inequality (11) holds because $\bar{a}(y) \cdot Q[y / x]$ can perform two consecutive restricted output actions not matchable by $\bar{a}(x) \cdot(P+(y / x] \cdot Q)$. The equality (12) holds as $\bar{a}(y) \cdot Q[y / x]$ can only be involved in a communication when restricted output and restricted update actions are banned.

Suppose $L \cap u=\emptyset$ and $x \neq y$. Let $A$ be $[x / x] \cdot\left(P_{1}+[y / x] \cdot Q\right)$ and $B$ be $[y / y] \cdot\left(P_{2}+[y / x] \cdot Q\right)$. Then

$$
\begin{aligned}
& A+B \not_{u} A+B+[y / x] \cdot Q \\
& A+B \approx_{L} A+B+[y / x] \cdot Q
\end{aligned}
$$

The inequality (13) is obvious. To understand (14) notice that if updates are banned then the component $[y / x] \cdot Q$ can be initiated when at least one of $x$ and $y$ is localized. If $x$ is localized then $[x / x] .\left(P_{1}+[y / x] . Q\right)$ can simulate $[y / x] \cdot Q$ and if $y$ is localized then it is for $[y / y] .\left(P_{2}+[y / x] . Q\right)$ to do the job.

Suppose $C$ is $[x / x] \cdot\left(P_{1}+\bar{a}[x] \cdot Q[x / y]\right)$ and $D$ is $[y / y] \cdot\left(P_{2}+[x / y] . Q\right)$. Then

$$
\begin{aligned}
& C+\bar{a}(y) \cdot(P+D) \not_{f_{o}} C+\bar{a}(y) \cdot(P+D)+\bar{a}[x] \cdot Q[x / y] \\
& C+\bar{a}(y) \cdot(P+D) \approx_{L} C+\bar{a}(y) \cdot(P+D)+\bar{a}[x] \cdot Q[x / y]
\end{aligned}
$$

when $L \cap(u \cup f o)=\emptyset$. Now (15) is clear. Justification of (16) is as follows: If the component $\bar{a}[x] \cdot Q[x / y]$ induces a restricted output (update) action, then $[x / x] .\left(P_{1}+\bar{a}[x] \cdot Q[x / y]\right)$ can simulate the action by performing first a tau action and then a restricted output (update). For example

$$
a[z]|(x)(C+\bar{a}(y) \cdot(P+D)+\bar{a}[x] \cdot Q[x / y]) \stackrel{(x / z]}{\longrightarrow} \mathbf{0}| Q[x / y][x / z]
$$

is simulated by $a[z]|(x)(C+\bar{a}(y) \cdot(P+D)) \stackrel{\tau}{\longrightarrow} a[z]|(x)\left(P_{1}+\bar{a}[x] \cdot Q[x / y]\right) \stackrel{(x / z]}{\longrightarrow}$ $\mathbf{0} \mid Q[x / y][x / z]$. If the component $\bar{a}[x] \cdot Q[x / y]$ is involved in a communication as in $(z) a[z] \cdot R|(C+\bar{a}(y) \cdot(P+D)+\bar{a}[x] \cdot Q[x / y]) \stackrel{\tau}{\longrightarrow} R[x / z]| Q[x / y]$. Then $\bar{a}(y) \cdot(P+$ $\left.[y / y] \cdot\left(P_{2}+[x / y] \cdot Q\right)\right)$ will put itself into action. The simulating sequence is:

$$
\begin{aligned}
(z) a[z] \cdot R \mid(C+\bar{a}(y) \cdot(P+D)) & \stackrel{\tau}{\longrightarrow}(y)\left(R[y / z] \mid\left(P+[y / y] \cdot\left(P_{2}+[x / y] \cdot Q\right)\right)\right) \\
& \stackrel{\tau}{\longrightarrow}(y)\left(R[y / z] \mid\left(P_{2}+[x / y] \cdot Q\right)\right) \\
& \stackrel{\tau}{\longrightarrow} R[x / z] \mid Q[x / y] .
\end{aligned}
$$

The reader is advised to play with these examples before moving on. 


\section{Open Bisimilarities}

The idea of open bisimilarity ([12]) is this: In order to show $P$ and $Q$ to be bisimilar, all one needs to consider are substitution instances of the pair. As a process contains only a finite number of names, it is usually enough to consider only a finite number of substitution instances. This is the basic reason for the effectiveness of open bisimilarity. The adjective "open" refers to the fact that in this approach the global names appearing in a process are treated very much like the free variables in, say, an open $\lambda$-term.

In this section we will define, for each $L \in \mathcal{L}$, an open bisimilarity that coincides with the $L$-bisimilarity. The proofs of coincidence not only support our definitions of open bisimulations for $\chi$-processes but also reveals much deeper properties of $L$-bisimilarities from the technical point of view.

Definition 6. (i) An open ro $\cup r u \cup i \cup f i \cup f o \cup u$-bisimulation is the same as an open $r o \cup r u \cup i \cup f i \cup f o \cup u$-relation.

(ii) An open ro $\cup r u \cup i \cup f i \cup$ fo-relation $\mathcal{R}$ is an open ro $\cup r u \cup i \cup f i \cup f_{o}$ bisimulation if the following properties hold for $P$ and $Q$ whenever $P \mathcal{R} Q$ :

- If $P \stackrel{[x / x]}{\longrightarrow} P^{\prime}$ then $Q^{\prime}$ exists such that either $Q \Longrightarrow_{x} Q^{\prime} \mathcal{R} P^{\prime}$ or some $y_{1}, \ldots, y_{n}$, $n \geq 1$, exist such that $Q \Longrightarrow x \stackrel{\left(y_{1} / x\right]}{\Longrightarrow} y_{1} \stackrel{\left(y_{2} / y_{1}\right]}{\Longrightarrow} y_{2} \ldots \stackrel{\left(y_{n} / y_{n-1}\right]}{\Longrightarrow} y_{n} Q^{\prime}$ and $Q^{\prime}\left[x / y_{n}\right] \mathcal{R} P^{\prime}$.

- If $P \stackrel{[y / x]}{\longrightarrow} P^{\prime}$, where $x \neq y$, then $Q^{\prime}$ exists such that either $Q \stackrel{[y / x]}{\Longrightarrow} Q^{\prime} \mathcal{R} P^{\prime}$ or both of the following properties hold:

- either $Q \stackrel{[x / x]}{\Longrightarrow} \stackrel{[y / x]}{\Longrightarrow} Q^{\prime} \mathcal{R} P^{\prime}$ or some $y_{1}, \ldots, y_{n}$, for $n \geq 1$, exist such that $Q \Longrightarrow x \stackrel{\left(y_{1} / x\right]}{\Longrightarrow} y_{1} \stackrel{\left(y_{2} / y_{1}\right]}{\longrightarrow} y_{2} \ldots \stackrel{\left(y_{n} / y_{n-1}\right]\left[y / y_{n}\right]}{\Longrightarrow} Q_{y_{n}} \stackrel{\mathcal{R}}{\longrightarrow} P^{\prime}$

- either $Q \stackrel{[y / y]}{\Longrightarrow} \stackrel{[y / x]}{\Longrightarrow} Q^{\prime} \mathcal{R} P^{\prime}$ or some $Q^{\prime \prime}, z_{1}, \ldots, z_{m}$, for $m \geq 1$, exist such

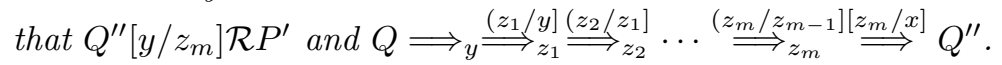

For each $L \in \mathcal{L}$, the open $L$-bisimilarity $\approx_{\text {open }}^{L}$ is the largest open L-bisimulation.

The above definition is not easy to digest. For motivations, the reader should check up the examples given in the previous section against the definition.

Theorem 7. Suppose $L \in \mathcal{L}$. Then $\approx_{\text {open }}^{L}$ coincides with $\approx_{L}$.

Proof. First we show that $\approx_{L} \subseteq \approx_{o p e n}^{L}$. Let $a$ be fresh throughout the proof.

(i) It follows from Definition 6 and Theorem 4 that $\approx_{r o \cup r u \cup i \cup f i \cup f o \cup u}$ is an open $r o \cup r u \cup i \cup f i \cup f o \cup u$-bisimulation.

(ii) Suppose $P \approx_{r o \cup r u \cup i \cup f i \cup f o} Q$ and $P \stackrel{[x / x]}{\longrightarrow} P^{\prime}$. Then $(x)(P \mid a[x]) \stackrel{\tau}{\longrightarrow} \stackrel{a x}{\longrightarrow}$ $P^{\prime} \mid \mathbf{0}$ must be matched up by $(x)(Q \mid a[x]) \stackrel{a x}{\Longrightarrow} Q^{\prime} \mid \mathbf{0}$ for some $Q^{\prime}$ such that $P^{\prime} \approx_{r o \cup r u \cup i \cup f i \cup f o} Q^{\prime}$. Using Lemma 2, one derives that either $Q \sigma \Longrightarrow_{x} Q^{\prime} \mathcal{R} P^{\prime}$ or some $y_{1}, \ldots, y_{n}$, for $n \geq 1$, exist such that $Q \sigma \Longrightarrow_{x} \stackrel{\left(y_{1} / x\right]}{\Longrightarrow} y_{1} \stackrel{\left(y_{2} / y_{1}\right]}{\Longrightarrow} y_{2} \ldots \stackrel{\left(y_{n} / y_{n-1}\right]}{\Longrightarrow} y_{n}{ }^{\prime}$ and $Q^{\prime}\left[x / y_{n}\right] \mathcal{R} P^{\prime}$.

If $P \stackrel{[y / x]}{\longrightarrow} P^{\prime}$ and $x \neq y$, then $(x)(P \mid a[x]) \stackrel{\tau}{\longrightarrow} P^{\prime} \mid a[y]$. To match up the reduction, there must exist some $Q^{\prime}$ such that $(x)(Q \mid a[x]) \stackrel{\tau}{\Longrightarrow} Q^{\prime} \mid a[y]$. According 
to Lemma 2, there are only three possibilities: either $Q \stackrel{[y / x]}{\Longrightarrow} Q^{\prime} \approx_{r o \cup r u \cup i \cup f i \cup f o}$ $P^{\prime}$, or $Q \stackrel{[x / x]}{\Longrightarrow} \stackrel{[y / x]}{\Longrightarrow} Q^{\prime} \approx_{r o \cup r u \cup i \cup f i \cup f o} P^{\prime}$, or $Q \Longrightarrow_{x} \stackrel{\left(y_{1} / x\right]}{\Longrightarrow} y_{1} \stackrel{\left(y_{2} / y_{1}\right]}{\Rightarrow} y_{2} \ldots \stackrel{\left(y_{n} / y_{n-1}\right]\left[y / y_{n}\right]}{\Longrightarrow}$ $Q^{\prime} \approx_{r o \cup r u \cup i \cup f i \cup f o} P^{\prime}$ for some $y_{1}, \ldots, y_{n}, n \geq 1$. If the first case is not possible, there must also exist some $Q^{\prime \prime}$ such that $(y)(Q \mid a[x]) \stackrel{(y / x]}{\Longrightarrow} Q^{\prime \prime} \mid a[y]$ matches up $(y)(P \mid a[x]) \stackrel{(y / x]}{\longrightarrow} P^{\prime} \mid a[y]$. Clearly $(y)(Q \mid a[x]) \stackrel{(y / x]}{\Longrightarrow} Q^{\prime \prime} \mid a[y]$ can be factorized as $(y)(Q \mid a[x]) \Longrightarrow(z)\left(Q_{1} \mid a[x]\right) \equiv(y)\left(Q_{1}[y / z] \mid a[x]\right) \stackrel{(y / x]}{\longrightarrow} Q_{2}\left|a[y] \Longrightarrow Q^{\prime \prime}\right| a[y] . \mathrm{By}$ Lemma 2, either $Q \sigma \stackrel{[y / y]}{\Longrightarrow} \stackrel{[y / x]}{\Longrightarrow} Q^{\prime} \mathcal{R} P^{\prime}$ or some $Q^{\prime \prime}, z_{1}, \ldots, z_{m}$, for $m \geq 1$, exist such that $Q^{\prime \prime}\left[y / z_{m}\right] \mathcal{R} P^{\prime}$ and $Q \sigma \Longrightarrow y \stackrel{\left(z_{1} / y\right]}{\Longrightarrow} z_{1}{\stackrel{\left(z_{2} / z_{1}\right]}{\longrightarrow}}_{z_{2}} \ldots \stackrel{\left(z_{m} / z_{m-1}\right]}{\Longrightarrow}{ }_{z_{m}} \stackrel{\left.z_{m} / x\right]}{\Longrightarrow} Q^{\prime \prime}$. Notice that $Q \sigma \stackrel{[y / x]}{\Longrightarrow} Q^{\prime} \mathcal{R} P^{\prime}$ is impossible by assumption.

The inclusion $\approx_{\text {open }}^{L} \subseteq \approx_{L}$ amounts to showing that, for each $L \in \mathcal{L}, \approx_{\text {open }}^{L}$ is an $L$-bisimulation.

With the help of the above theorem we can now define $L$-congruences by exploiting the explicit requirement in the definition of open $L$-bisimilarities.

Definition 8. Two processes $P$ and $Q$ are L-congruent, written $P={ }_{L} Q$, if $P \approx_{\text {open }}^{L} Q$ and, for every substitution $\sigma$ and every $\phi \in r u \cup u \cup\{\tau\}, P \sigma \stackrel{\phi}{\longrightarrow} P^{\prime}$ must be matched up by a nonempty sequence of actions from $Q$ and vice versa.

So for instance, if $P={ }_{r o \cup r u \cup i \cup f i \cup f o} Q$ then it should not be the case that the only way $Q$ can simulate $P \stackrel{[x / x]}{\longrightarrow} P^{\prime}$ is by the vacuous action $Q \Longrightarrow Q$.

\section{Prefix Laws}

Let $A S$ be the system given in Figure 2 plus the equivalence rules, the congruence rules and the following expansion law, in which $\pi$ and $\gamma$ range over $\{\tau\} \cup\{\alpha[x],[y / x] \mid x, y \in \mathcal{N}\}:$

$$
\begin{aligned}
P \mid Q= & \sum_{i \in I}\left[M_{i}\right](\boldsymbol{x}) \pi_{i} \cdot\left(P_{i} \mid Q\right)+\sum_{\gamma_{j}=\overline{b_{j}}\left[y_{j}\right]}^{\pi_{i}=a_{i}\left[x_{i}\right]}\left[M_{i}\right]\left[N_{j}\right](\boldsymbol{x})(\boldsymbol{y})\left[a_{i}=b_{j}\right]\left[y_{j} / x_{i}\right] .\left(P_{i} \mid Q_{j}\right) \\
& +\sum_{j \in J}\left[N_{j}\right](\boldsymbol{y}) \gamma_{j} \cdot\left(P \mid Q_{j}\right)+\sum_{\gamma_{j}=b_{j}\left[y_{j}\right]}^{\pi_{i}=\overline{a_{i}}\left[x_{i}\right]}\left[M_{i}\right]\left[N_{j}\right](\boldsymbol{x})(\boldsymbol{y})\left[a_{i}=b_{j}\right]\left[x_{i} / y_{j}\right] .\left(P_{i} \mid Q_{j}\right)
\end{aligned}
$$

where $P$ is $\sum_{i \in I}\left[M_{i}\right](\boldsymbol{x}) \pi_{i} . P_{i}, Q$ is $\sum_{j \in J}\left[N_{j}\right](\boldsymbol{y}) \gamma_{j} \cdot Q_{j}$ and $\{\boldsymbol{x}\} \cap\{\boldsymbol{y}\}=\emptyset$. The second component in the right hand of the above equality captures the idea that whenever $\pi_{i}$ is of the form $a_{i}\left[x_{i}\right]$ for some $i \in I$ and $\gamma_{j}$ is of the form $\overline{b_{j}}\left[y_{j}\right]$ for some $j \in J$ then there is a summand $\left[M_{i}\right]\left[N_{j}\right](\boldsymbol{x})(\boldsymbol{y})\left[a_{i}=b_{j}\right]\left[y_{j} / x_{i}\right] .\left(P_{i} \mid Q_{j}\right)$.

We will write $A S \cup\left\{R_{1}, \ldots, R_{n}\right\} \vdash P=Q$ to mean that the equality $P=Q$ is derivable from the axioms and rules of $A S$ together with axioms and rules $R_{1}, \ldots, R_{n}$. When no confusion arises, we simply write $P=Q$. We will also write $P \stackrel{R}{=} Q$ to indicate that $R$ is the major axiom applied to derive $P=Q$. 


\begin{tabular}{|c|c|c|}
\hline L1 & $(x) \mathbf{0}=\mathbf{0}$ & \\
\hline $\mathrm{L} 2$ & $(x) \alpha[y] . P=\mathbf{0}$ & $x \in\{\alpha, \bar{\alpha}\}$ \\
\hline L3 & $(x) \alpha[y] \cdot P=\alpha[y] \cdot(x) P$ & $x \notin\{y, \alpha, \bar{\alpha}\}$ \\
\hline L4 & $(x)(y) P=(y)(x) P$ & \\
\hline L5 & $(x)[y=z] P=[y=z](x) P$ & $x \notin\{y, z\}$ \\
\hline L6 & $(x)(P+Q)=(x) P+(x) Q$ & \\
\hline L7 & $(x)[x=y] P=\mathbf{0}$ & \\
\hline L8 & $(x)[y / x] \cdot P=\tau \cdot P[y / x]$ & \\
\hline L9 & $(x)[y / z] \cdot P=[y / z] \cdot(x) P$ & $x \notin\{y, z\}$ \\
\hline L10 & $(x) \tau . P=\tau .(x) P$ & \\
\hline M1 & {$[M] P=[N] P$} & if $M \Leftrightarrow N$ \\
\hline M2 & {$[x=y] P=[x=y] P[y / x]$} & \\
\hline M3 & {$[x=y](P+Q)=[x=y] P+[x=y] Q$} & \\
\hline S1 & $P+\mathbf{0}=P$ & \\
\hline S2 & $P+Q=Q+P$ & \\
\hline S3 & $P+(Q+R)=(P+Q)+R$ & \\
\hline S4 & {$[x=y] P+P=P$} & \\
\hline U1 & {$[y / x] \cdot P=[y / x] \cdot[x=y] P$} & \\
\hline MD1 & {$[x=y] .0=0$} & derivable from S1 and S4 \\
\hline MD2 & {$[x=x] . P=P$} & derivable from M1 \\
\hline MD3 & {$[M] P=[M]\left(P \sigma_{M}\right)$} & derivable from $\mathrm{M} 2$ \\
\hline SD1 & $P+P=P$ & derivable from MD2 and $\mathrm{S} 4$ \\
\hline SD2 & {$[M] P+P=P$} & derivable from S-rules \\
\hline UD1 & {$[y / x] \cdot P=[y / x] \cdot P[y / x]$} & derivable from $\mathrm{U} 1$ and $\mathrm{M} 2$ \\
\hline
\end{tabular}

Fig. 2. Axioms for Strong Bisimilarity on Chi Processes

In $A S$ every process $P$ can be converted to a normal form process of the following shape: $\sum_{i \in I_{1}}\left[M_{i}\right] \alpha_{i}\left[x_{i}\right] \cdot P_{i}+\sum_{i \in I_{2}}\left[M_{i}\right] \alpha_{i}(x) \cdot P_{i}+\sum_{i \in I_{3}}\left[M_{i}\right]\left[y_{i} / x_{i}\right] \cdot P_{i}+$ $\sum_{i \in I_{4}}\left[M_{i}\right]\left(y / x_{i}\right] . P_{i}+\sum_{i \in I_{5}}\left[M_{i}\right] \tau . P_{i}$, in which neither $x$ nor $y$ appears global in $P$ and $P_{i}$ is in normal form for each $i \in I_{1} \cup I_{2} \cup I_{3} \cup I_{4} \cup I_{5}$. Here $I_{1}, I_{2}, I_{3}, I_{4}$ and $I_{5}$ are pairwise disjoint finite indexing sets.

$A S$ is sound and complete for strong bisimilarity whose definition we omit, but see [10]. In order to lift $A S$ to complete systems for $L$-congruences, we propose 17 axioms as given in Figure 3. We call them prefix laws as they are mainly dealing with prefix combinators. The first three are the well-known tau laws. We have seen axioms $\mathrm{P} 4$ and $\mathrm{P} 14$ in [3]. The other twelve axioms are new. In what follows, $A S_{\tau}$ denotes $A S \cup\{P 1, P 2, P 3\}$.

\section{Saturation Property}

In the standard proof of completeness theorem for weak congruence on finite CCS processes, one verifies first that every normal form process is provably equal to a saturated normal form process using the three tau laws. Recall that a process $P$ is saturated if, for every $\alpha, P \stackrel{\alpha}{\longrightarrow} P^{\prime}$ whenever $P \stackrel{\alpha}{\longrightarrow} P^{\prime}$. Now if $P$ and $Q$ are weakly congruent saturated normal form processes and $P \stackrel{\alpha}{\longrightarrow} P^{\prime}$ then 


\begin{tabular}{|c|c|}
\hline & $\delta . \tau . P=\delta . P$ \\
\hline $\mathrm{P} 2$ & $P+\tau . P=\tau . P$ \\
\hline P3 & $\delta .(P+\tau . Q)=\delta .(P+\tau \cdot Q)+\delta . Q$ \\
\hline $\mathrm{P} 4$ & $a(y) \cdot(P+[y / x] \cdot Q)=a(y) \cdot(P+[y / x] \cdot Q)+a[x] \cdot Q[x / y]$ \\
\hline $\mathrm{P} 5$ & $(y / x] . P+\tau . P[x / y]=\tau . P[x / y]$ \\
\hline P6 & $(y / x] \cdot(P+(z / y] \cdot Q)=(y / x] \cdot(P+(z / y] \cdot Q)+(z / x] \cdot Q[z / y]$ \\
\hline P7 & $\bar{a}(x) \cdot(P+(y / x] \cdot Q)=\bar{a}(x) \cdot(P+(y / x] \cdot Q)+\bar{a}(y) \cdot Q[y / x]$ \\
\hline $\mathrm{P} 8$ & $A+B=A+B+[y / x] \cdot Q$ \\
\hline P9 & $C+\bar{a}(y) \cdot(P+D)=C+\bar{a}(y) \cdot(P+D)+\bar{a}[x] \cdot Q[x / y]$ \\
\hline P10 & $E+a(y) \cdot(P+F)=E+a(y) \cdot(P+F)+a[x] \cdot Q[x / y]$ \\
\hline P11 & $G+a(y) \cdot(P+F)=G+a(y) \cdot(P+F)+a[x] \cdot Q[x / y]$ \\
\hline $\mathrm{P} 12$ & $(y / x] \cdot(P+[z / y] \cdot Q)=(y / x] \cdot(P+[z / y] \cdot Q)+[z / x] \cdot Q[z / y]$ \\
\hline P13 & $(y / x] \cdot(P+a[y] \cdot Q)=(y / x] \cdot(P+a[y] \cdot Q)+a[x] \cdot Q[x / y]$ \\
\hline $\mathrm{P} 14$ & $\bar{a}(x) \cdot(P+[y / x] \cdot Q)=\bar{a}(x) \cdot(P+[y / x] \cdot Q)+\bar{a}[y] \cdot Q[y / x]$ \\
\hline P15 & $(y / x] \cdot P+[x / x] \cdot P[x / y]=[x / x] \cdot P[x / y]$ \\
\hline $\mathrm{P} 16$ & $(y / x] \cdot P+[x / x] \cdot P[x / y]=(y / x] \cdot P$ \\
\hline $\mathrm{P} 17$ & {$[x / x] . P+\tau . P=\tau . P$} \\
\hline \multicolumn{2}{|r|}{$\begin{array}{l}\text { In } \mathrm{P} 8, A \equiv[x / x] \cdot\left(P_{1}+[y / x] \cdot Q\right) \text { and } B \equiv[y / y] \cdot\left(P_{2}+[y / x] \cdot Q\right) . \\
\text { In P9, } C \equiv[x / x] \cdot\left(P_{1}+\bar{a}[x] \cdot Q[x / y]\right) \text { and } D \equiv[y / y] \cdot\left(P_{2}+[x / y] \cdot Q\right) \text {. } \\
\text { In P10, } E \equiv[x / x] \cdot\left(P_{1}+a[x] \cdot Q[x / y]\right) \text { and } F \equiv[y / y] \cdot\left(P_{2}+[y / x] \cdot Q\right) \\
\text { In P11, } G \equiv[x / x] \cdot\left(P_{1}+a(y) \cdot\left(Q_{1}+[x / x] \cdot\left(Q_{2}+[y / x] \cdot Q\right)\right)\right) \text { and } \\
F \equiv[y / y] \cdot\left(P_{2}+[y / x] \cdot Q\right) \text {. }\end{array}$} \\
\hline
\end{tabular}

Fig. 3. The Prefix Laws

$Q \stackrel{\alpha}{\Longrightarrow} Q^{\prime}$ for some $Q^{\prime}$ such that $Q^{\prime} \approx P^{\prime}$, where $\approx$ denotes weak bisimilarity. By saturation, $Q \stackrel{\alpha}{\longrightarrow} Q^{\prime}$ and therefore $\alpha . Q^{\prime}$ is a summand of $Q$. If, and this is a nontrivial if, we can deduce by induction hypothesis that $\alpha . P^{\prime}$ is provably equal to $\alpha \cdot Q^{\prime}$, then we can conclude that every summand of $P$ is provably equal to a summand of $Q$, and vice versa. This gives us the required completeness.

If one is focusing only on completeness proof, then the notion of saturated process is a distraction. All one really needs is the following saturation property:

If $P \stackrel{\alpha}{\Longrightarrow} P^{\prime}$ for normal form $P$, then $P$ and $P+\alpha \cdot P^{\prime}$ are provably equal.

This is the first of the two crucial properties a completeness proof rests upon. Another one is to be discussed in next section. These properties suffice to establish the following absorption property:

If two normal form processes $P$ and $Q$ are congruent then $P+Q$ is provably equal to $P$.

Of course, under the same assumption, $P+Q$ is also provably equal to $Q$. Hence the completeness.

In $\chi$-calculus, a basic saturation lemma would say that $P$ and $P+\delta . P^{\prime}$ are provably equal whenever $P \stackrel{\delta}{\Longrightarrow} P^{\prime}$ for normal form process $P$. But this is far from sufficient. Suppose $P={ }_{r o \cup r u \cup i \cup f i \cup f o} Q$ for normal form processes $P$ and $Q$. 
Suppose further that $[x / x] . P^{\prime}$ is a summand of $P$. Then some $Q^{\prime}$ must exist such that either $P^{\prime} \approx_{r o \cup r u \cup i \cup f i \cup f o} Q^{\prime}$ and $Q \Longrightarrow_{x} Q^{\prime}$ or $P^{\prime} \approx_{r o \cup r u \cup i \cup f i \cup f o} Q^{\prime}\left[x / y_{n}\right]$ and some $y_{1}, \ldots, y_{n}$, for $n \geq 1$, exist such that $\left.Q \Longrightarrow x \stackrel{\left(y_{1} / x\right]}{\longrightarrow} y_{1} \stackrel{\left(y_{2} / y_{1}\right]}{\longrightarrow} y_{2} \ldots \stackrel{\left(y_{n} / y_{n-1}\right]}{\Longrightarrow} y_{n}\right]$ $Q^{\prime}$. In the former case we have, by the basic saturation lemma, that $Q$ is provably equal to $Q+[x / x] . Q^{\prime}$. In the latter case we would also like to say the same. But it no longer follows from the basic saturation lemma. Extra axiom are necessary to derive the equality $Q=Q+[x / x] \cdot Q^{\prime}$.

Lemma 9. Suppose $Q$ is in normal form. Then the following properties hold:

(1) If $Q \sigma_{M} \stackrel{\tau}{\Longrightarrow} Q^{\prime}$ then $A S_{\tau} \vdash Q=Q+[M] \tau \cdot Q^{\prime}$.

(2) If $Q \sigma_{M} \stackrel{\alpha[x]}{\Longrightarrow} Q^{\prime}$ then $A S_{\tau} \vdash Q=Q+[M] \alpha[x] \cdot Q^{\prime}$.

(3) If $z \notin g n(Q) \cup n(M)$ and $Q \sigma_{M} \stackrel{a z}{=} Q^{\prime}$ then $A S_{\tau} \vdash Q=Q+[M] a(z) \cdot Q^{\prime}$.

(4) If $z \notin g n(Q) \cup n(M)$ and $Q \sigma_{M} \stackrel{\bar{a}(z)}{\Longrightarrow} Q^{\prime}$ then $A S_{\tau} \vdash Q=Q+[M] \bar{a}(z) \cdot Q^{\prime}$.

(5) If $Q \sigma_{M} \stackrel{[y / x]}{\Longrightarrow} Q^{\prime}$ then $A S_{\tau} \vdash Q=Q+[M][y / x] . Q^{\prime}$.

(6) If $y \notin g n(Q) \cup n(M)$ and $Q \sigma_{M} \stackrel{(y / x]}{\Longrightarrow} Q^{\prime}$ then $A S_{\tau} \vdash Q=Q+[M](y / x] . Q^{\prime}$.

(7) If $Q \sigma_{M} \stackrel{\tau}{\Longrightarrow} Q_{x}^{\prime}$ or $Q \sigma_{M} \stackrel{[x / x]}{\Longrightarrow} Q^{\prime}$ then $A S_{\tau} \cup\{P 8, P 17\} \vdash Q=Q+$ $[M][x / x] \cdot Q^{\prime}$.

(8) If $Q \sigma_{M} \Longrightarrow x \stackrel{\left(y_{1} / x\right]}{\Longrightarrow} y_{1} \stackrel{\left(y_{2} / y_{1}\right]}{\Longrightarrow} y_{2} \ldots \stackrel{\left(y_{n} / y_{n-1}\right]}{\Longrightarrow} y_{n} Q^{\prime}$ then $Q=Q+[M][x / x] \cdot Q^{\prime}\left[x / y_{n}\right]$ is provable in the system $A S_{\tau} \cup\{P 8, P 16, P 17\}$.

Proof. (8) Suppose $Q \sigma_{M} \Longrightarrow_{x} Q_{1} \stackrel{\left(y_{1} / x\right]}{\Longrightarrow} Q_{2} \cdots Q_{2 n-1} \stackrel{\left(y_{n} / y_{n-1}\right]}{\Longrightarrow} Q_{2 n} \Longrightarrow y_{n} Q^{\prime}$. Now $x \notin Q_{2}, x \notin Q_{3} ; x, y_{1} \notin Q_{4}, x, y_{1} \notin Q_{5} ; \ldots ; x, y_{1}, \ldots, y_{n-2} \notin Q_{2 n-2}$, $x, y_{1}, \ldots, y_{n-2} \notin Q_{2 n-1} ; x, y_{1}, \ldots, y_{n-1} \notin Q_{2 n}, x, y_{1}, \ldots, y_{n-1} \notin Q^{\prime}$. Therefore $Q_{2 i-2}\left[x / y_{i-1}\right] \Longrightarrow Q_{2 i-1}\left[x / y_{i-1}\right] \stackrel{\left(y_{i} / x\right]}{\Longrightarrow} Q_{2 i}$, for $2 \leq i \leq n$, and $Q_{2 n}\left[x / y_{n}\right] \Longrightarrow_{x}$ $Q^{\prime}\left[x / y_{n}\right]$ by Lemma 1 . With these observations one obtains the following inference, assuming $Q \sigma_{M} \Longrightarrow_{x} Q_{1}$ is not vacuous:

$$
\begin{aligned}
Q & =Q+[M][x / x] \cdot\left(Q_{1}+\left(y_{1} / x\right] \cdot Q_{2}\right) \\
& \stackrel{P 16}{=} Q+[M][x / x] \cdot\left(Q_{1}+\left(y_{1} / x\right] \cdot Q_{2}+[x / x] \cdot Q_{2}\left[x / y_{1}\right]\right) \\
& =Q+[M]\left([x / x] \cdot\left(Q_{1}+\left(y_{1} / x\right] \cdot Q_{2}+[x / x] \cdot Q_{2}\left[x / y_{1}\right]\right)+[x / x] \cdot Q_{2}\left[x / y_{1}\right]\right) \\
& =Q+[M][x / x] \cdot Q_{2}\left[x / y_{1}\right] \\
& \vdots \\
& =Q+[M][x / x] \cdot Q^{\prime}\left[x / y_{n}\right]
\end{aligned}
$$

where the first equality holds by (6) and (7) of this lemma; the third equality is a consequence of $\mathrm{P} 8$. If $Q \sigma_{M} \Longrightarrow_{x} Q_{1}$ is vacuous then

$$
\begin{aligned}
Q & =Q+[M]\left(y_{1} / x\right] \cdot Q_{2} \\
& \stackrel{P 16}{=} Q+[M]\left(\left(y_{1} / x\right] \cdot Q_{2}+[x / x] \cdot Q_{2}\left[x / y_{1}\right]\right) \\
& =Q+[M][x / x] \cdot Q_{2}\left[x / y_{1}\right] .
\end{aligned}
$$

So the previous inference is valid anyway. 


\begin{tabular}{|ll|}
\hline $\operatorname{Pr} 1$ & $\tau . P=\tau .\left(P+\sum_{i \in I}\left[M_{i}\right] \tau \cdot P\right)$ \\
$\operatorname{Pr} 2$ & $\tau . P=\tau .\left(P+\sum_{i \in I_{1}}\left[M_{i}\right] \tau \cdot P+\sum_{i \in I_{2}}\left[M_{i}\right]\left(w / x_{i}\right] . P\right)$ \\
$\operatorname{Pr} 3$ & $\tau . P=\tau .\left(P+\sum_{i \in I_{1}}\left[M_{i}\right] \tau \cdot P+\sum_{i \in I_{2}}\left[M_{i}\right]\left[x_{i} / x_{i}\right] . P\right)$ \\
$\operatorname{Pr} 4$ & $\tau . P=\tau \cdot\left(P+\sum_{i \in I_{1}}\left[M_{i}\right] \tau \cdot P+\sum_{i \in I_{2}}\left[M_{i}\right]\left(w / x_{i}\right] . P+\sum_{i \in I_{3}}\left[M_{i}\right]\left[x_{i} / x_{i}\right] . P\right)$ \\
\hline In the above axioms, $w$ is fresh, $I, I_{1}, I_{2}, I_{3}$ are finite indexing sets. \\
\hline
\end{tabular}

Fig. 4. The Promotion Axioms

\section{Promotion Property}

In the proof of completeness theorem for weak congruence in CCS, the following result, due to Hennessy, plays a crucial role:

If $P \approx Q$ then either $\tau \cdot P=Q$ or $P=Q$ or $P=\tau . Q$.

Here $\approx$ is the weak bisimilarity and $=$ is the congruence induced by $\approx$. In the proof of the completeness theorem by induction, Hennessy Lemma helps to lift $P \approx Q$ to either $\tau \cdot P=Q$ or $P=Q$ or $P=\tau \cdot Q$, thus allowing the induction hypothesis to apply. In $\pi$-calculus however Hennessy Lemma does not hold! For a counter example, consider the following three propositions

$$
\begin{aligned}
\tau \cdot(\bar{a} x+[x=y] \tau \cdot \bar{a} y) & =\bar{a} x \\
\bar{a} x+[x=y] \tau \cdot \bar{a} y & =\bar{a} x \\
\bar{a} x+[x=y] \tau \cdot \bar{a} y & =\tau \cdot \bar{a} x
\end{aligned}
$$

None of them holds although $\bar{a} x+[x=y] \tau \cdot \bar{a} y \approx \bar{a} x$ is true. This example explains the reason why nobody has given a proof that Sangiorgi's system ([12]) together with Milner's tau laws constitute a complete system for weak open congruence. We believe that the resulting system is not capable of establishing the equality $\tau \cdot(\bar{a} x+[x=y] \tau \cdot \bar{a} y)=\tau \cdot \bar{a} x$.

The purpose of this section is to present our solution. The motivation comes from a careful examination of the role of Hennessy Lemma in CCS. What it really comes down to is the following promotion property:

If $P \approx Q$ for normal form processes $P$ and $Q$ then $\tau . P=\tau$. $Q$ is provable.

Motivated by this observation, we introduce four additional axioms as given in Figure 4. In the presence of a suitable set of prefix laws, these four axioms are capable of lifting $A S$ to a complete system for an $L$-congruence. For this reason we call them promotion axioms. Clearly both Pr2 and Pr3 subsume Pr1 and are subsumed by $\operatorname{Pr} 4$.

Theorem 10. Suppose $P$ and $Q$ are in normal form.

(i) If $P \approx_{r o \cup r u \cup i \cup f i \cup f o \cup u} Q$ then $A S_{\tau} \cup\{\operatorname{Pr} 1\} \vdash \tau . P=\tau . Q$.

(ii) If $P \approx_{r o \cup r u \cup i \cup f i \cup f o} Q$ then $A S_{\tau} \cup\{P 8, P 16, P 17, P r 3\} \vdash \tau . P=\tau . Q$. 
Proof. We prove (ii) only. Suppose $P \approx_{r o \cup r u \cup i \cup f i \cup f o} Q$ for normal form processes $P$ and $Q$. The proof is carried out by induction on the sum of the depths of $P$ and $Q$. Let $P$ be of the form $\sum_{i \in I_{1}}\left[M_{i}\right] \alpha_{i}\left[x_{i}\right] . P_{i}+\sum_{i \in I_{2}}\left[M_{i}\right] \alpha_{i}(x) . P_{i}+$ $\sum_{i \in I_{3}}\left[M_{i}\right]\left[y_{i} / x_{i}\right] . P_{i}+\sum_{i \in I_{4}}\left[M_{i}\right]\left(y / x_{i}\right] . P_{i}+\sum_{i \in I_{5}}\left[M_{i}\right] \tau \cdot P_{i}$ and $Q$ be of the form $\sum_{j \in J_{1}}\left[N_{j}\right] \alpha_{j}\left[x_{j}\right] \cdot Q_{j}+\sum_{j \in J_{2}}\left[N_{j}\right] \alpha_{j}(x) \cdot Q_{j}+\sum_{j \in J_{3}}\left[N_{j}\right]\left[y_{j} / x_{j}\right] \cdot Q_{j}+\sum_{j \in J_{4}}\left[N_{j}\right](y /$
$\left.x_{j}\right] \cdot Q_{j}+\sum_{j \in J_{5}}\left[N_{j}\right] \tau \cdot Q_{j}$.

Suppose, for $i \in I_{3}, y_{i} \sigma_{M_{i}}=x_{i} \sigma_{M_{i}}$ and $\left(\left[M_{i}\right]\left[y_{i} / x_{i}\right] . P_{i}\right) \sigma_{M_{i}} \stackrel{\left[y_{i} \sigma_{M_{i}} / x_{i} \sigma_{M_{i}}\right]}{\longrightarrow}$ $P_{i} \sigma_{M_{i}}$ can only be matched up vacuously by $Q \sigma_{M_{i}}$. Then $A S_{\tau} \cup\{P 8, P 16, P 17\} \vdash$ $\tau . P_{i} \sigma_{M_{i}}=\tau \cdot Q \sigma_{M_{i}}$ by induction hypothesis. It follows that

$$
A S_{\tau} \cup\{P 8, P 16, P 17\} \vdash\left[M_{i}\right]\left[y_{i} / x_{i}\right] . P_{i}=\left[M_{i}\right]\left[x_{i} / x_{i}\right] . Q .
$$

Suppose, for some $i \in I_{3}, y_{i} \sigma_{M_{i}} \neq x_{i} \sigma_{M_{i}}$. Using Lemma 9 and axiom P8, one can show that $A S_{\tau} \cup\{P 8, P 16, P 17\} \vdash\left[M_{i}\right]\left[y_{i} / x_{i}\right] . P_{i}+Q=Q$.

Similarly one shows that some $I^{\prime} \subseteq I_{5}$ exists such that the equality $\left[M_{i}\right] \tau . P_{i}=$ $\left[M_{i}\right] \tau . Q$ is provable in $A S_{\tau} \cup\{P 8, \bar{P} 16, P 17\}$ for each $i \in I^{\prime}$ and that $A S_{\tau} \cup$ $\{P 8, P 16, P 17\} \vdash\left[M_{i}\right] \tau . P_{i}+Q=Q$ for each $i \in I_{5} \backslash I^{\prime}$.

It is also clear that $A S_{\tau} \cup\{P 8, P 16, P 17\} \vdash\left[M_{i}\right] \alpha_{i}\left[x_{i}\right] . P_{i}+Q=Q$, respectively $A S_{\tau} \cup\{P 8, P 16, P 17\} \vdash\left[M_{i}\right] \alpha_{i}(x) . P_{i}+Q=Q, A S_{\tau} \cup\{P 8, P 16, P 17\} \vdash$ $\left[M_{i}\right]\left(y / x_{i}\right] . P_{i}+Q=Q$, if $\left[M_{i}\right] \alpha_{i}\left[x_{i}\right] . P_{i}$, respectively $\left[M_{i}\right] \alpha_{i}(x) . P_{i},\left[M_{i}\right]\left(y / x_{i}\right] . P_{i}$, is a summand of $P$.

We can now conclude that $P+Q=Q+\Sigma_{i \in I^{\prime}}\left[M_{i}\right] \tau . Q+\Sigma_{i \in I}\left[M_{i}\right]\left[x_{i} / x_{i}\right] . Q$ is provable in the system $A S_{\tau} \cup\{P 8, P 16, P 17\}$ for some $I \subseteq I_{3}$ and $I^{\prime} \subseteq I_{5}$. Now $\tau . Q=\tau .\left(Q+\Sigma_{i \in I^{\prime}}\left[M_{i}\right] \tau . Q+\Sigma_{i \in I}\left[M_{i}\right]\left[x_{i} / x_{i}\right] . Q\right)$ by Pr3. It follows that $A S_{\tau} \cup\{P 8, P 16, P 17, \operatorname{Pr} 3\} \vdash \tau .(P+Q)=\tau . Q$.

Symmetrically $A S_{\tau} \cup\{P 8, P 16, P 17, \operatorname{Pr} 3\} \vdash \tau .(P+Q)=\tau . P$. Therefore $A S_{\tau} \cup\{P 8, P 16, P 17, \operatorname{Pr} 3\} \vdash \tau . P=\tau . Q$.

\section{Completeness Theorem}

Having done all the preparations, we finally come to the completeness theorem. Its proof is so similar to the proof of the promotion lemma reported in the previous section as to render any reiteration redundant.

Theorem 11. (i) $A S_{\tau} \cup\{\operatorname{Pr} 1\}$ is sound and complete for $={ }_{r o \cup r u \cup i \cup f i \cup f o \cup u}$. (ii) $A S_{\tau} \cup\{P 8, P 16, P 17, \operatorname{Pr} 3\}$ is sound and complete for $={ }_{r o \cup r u \cup i \cup f i \cup f o}$.

Proof. Let's see how (ii) is proved. The soundness is easy. Suppose both $P$ and $Q$ are in normal form and $P={ }_{r o \cup r u \cup i \cup f i \cup f o} Q$. Using (ii) of Theorem 10 and its proof, one concludes that $P+Q=Q+\Sigma_{i \in I^{\prime}}\left[M_{i}\right] \tau . Q+\Sigma_{i \in I}\left[M_{i}\right]\left[x_{i} / x_{i}\right] . Q$ is provable in the system $A S_{\tau} \cup\{P 8, P 16, P 17\}$ for some $I \subseteq I_{3}$ and $I^{\prime} \subseteq I_{5}$. But $Q$ must be able to simulate a first move of $P$ by a nonempty sequence of moves. This implies that both $I$ and $I^{\prime}$ are empty. It follows that $P+Q=Q$ is provable in $A S_{\tau} \cup\{P 8, P 16, P 17, \operatorname{Pr} 3\}$. Similarly $P+Q=P$ is provable in $A S_{\tau} \cup\{P 8, P 16, P 17, \operatorname{Pr} 3\}$. Hence $A S_{\tau} \cup\{P 8, P 16, P 17, \operatorname{Pr} 3\} \vdash P=Q$. 


\begin{tabular}{|c|c|c|}
\hline Congruence & \multicolumn{2}{|c|}{ Axioms in Addition to $A S \cup\{\mathrm{P} 1, \mathrm{P} 2, \mathrm{P} 3\}$} \\
\hline$=r o \cup r u \cup i \cup f i \cup f o \cup u$ & & Pr1 \\
\hline$={ }_{r o \cup r u \cup i \cup f o \cup u}$ & $\mathrm{P} 4$ & Pr1 \\
\hline$={ }_{r o \cup i \cup f i \cup f o \cup u}$ & $\mathrm{P} 5, \mathrm{P} 6, \mathrm{P} 15$ & Pr2 \\
\hline$=i \cup f i \cup f o \cup u$ & $\mathrm{P} 5, \mathrm{P} 7, \mathrm{P} 15$ & $\operatorname{Pr} 2$ \\
\hline$={ }_{r o \cup i \cup f o \cup u}$ & $\mathrm{P} 4, \mathrm{P} 5, \mathrm{P} 6, \mathrm{P} 15$ & Pr2 \\
\hline$={ }_{i \cup f o \cup u}$ & $\mathrm{P} 4, \mathrm{P} 5, \mathrm{P} 7, \mathrm{P} 15$ & Pr2 \\
\hline$={ }_{r o \cup r u \cup i \cup f i \cup f o}$ & $\mathrm{P} 8, \mathrm{P} 16, \mathrm{P} 17$ & Pr3 \\
\hline$={ }_{r o \cup r u \cup i \cup f i}$ & $\mathrm{P} 8, \mathrm{P} 9, \mathrm{P} 16, \mathrm{P} 17$ & Pr3 \\
\hline$=r o \cup r u \cup i \cup f o$ & $\mathrm{P} 4, \mathrm{P} 8, \mathrm{P} 10, \mathrm{P} 11, \mathrm{P} 16, \mathrm{P} 17$ & Pr3 \\
\hline$={ }_{r o \cup r u \cup i}$ & $\mathrm{P} 4, \mathrm{P} 8, \mathrm{P} 9, \mathrm{P} 10, \mathrm{P} 11, \mathrm{P} 16, \mathrm{P} 17$ & Pr3 \\
\hline$=r o \cup i \cup f i \cup f o$ & $\mathrm{P} 5, \mathrm{P} 6, \mathrm{P} 12, \mathrm{P} 15, \mathrm{P} 16$ & Pr4 \\
\hline$={ }_{i \cup f i \cup f o}$ & $\mathrm{P} 5, \mathrm{P} 7, \mathrm{P} 12, \mathrm{P} 15, \mathrm{P} 16$ & Pr4 \\
\hline$=r o \cup i \cup f o$ & $\mathrm{P} 4, \mathrm{P} 5, \mathrm{P} 6, \mathrm{P} 13, \mathrm{P} 15, \mathrm{P} 16$ & Pr4 \\
\hline$={ }_{i \cup f o}$ & $\mathrm{P} 4, \mathrm{P} 5, \mathrm{P} 7, \mathrm{P} 13, \mathrm{P} 15, \mathrm{P} 16$ & Pr4 \\
\hline$={ }_{r o \cup i \cup f i}$ & $\mathrm{P} 5, \mathrm{P} 6, \mathrm{P} 9, \mathrm{P} 12, \mathrm{P} 15, \mathrm{P} 16$ & Pr4 \\
\hline$={ }_{r o \cup i}$ & $\mathrm{P} 4, \mathrm{P} 5, \mathrm{P} 6, \mathrm{P} 9, \mathrm{P} 13, \mathrm{P} 15, \mathrm{P} 16$ & Pr4 \\
\hline$={ }_{i \cup f i}$ & $\mathrm{P} 5, \mathrm{P} 7, \mathrm{P} 9, \mathrm{P} 14, \mathrm{P} 15, \mathrm{P} 16$ & Pr4 \\
\hline$={ }_{i}$ & $\mathrm{P} 4, \mathrm{P} 5, \mathrm{P} 7, \mathrm{P} 9, \mathrm{P} 13, \mathrm{P} 14, \mathrm{P} 15, \mathrm{P} 16$ & $\operatorname{Pr} 4$ \\
\hline
\end{tabular}

Fig. 5. Summary of the 18 Completeness Systems

\section{Concluding Remarks}

The work reported in this paper consists of two parts. The first part is a continuation of the study of $L$-bisimilarities on asymmetric $\chi$-processes initiated in [3]. The result of this investigation is a finer description of $L$-bisimilarities in terms of open $L$-bisimilarities. This alternative view leads immediately to the explicit definition of the largest congruence relation, the $L$-congruence, contained in an $L$-bisimilarity. Building upon the first part, the second part explains a streamlined approach to derive complete systems for $L$-congruences. In addition to the axioms and rules for strong bisimilarity, 21 axioms are proposed. It is shown that these are enough to lift a complete system for the strong bisimilarity to complete systems for $L$-congruences. Due to space restriction, the paper discusses only two $L$-bisimilarities. The definitions of the other sixteen open $L$-bisimilarities fit into the pattern of Definition 6. Most of them are even more complex. It should be emphasized that these definitions are not simply a matter of putting things together. In Figure 5 all the 18 complete systems for $L$-congruences are given.

It can be easily shown that the top element of the bisimulation lattice coincides with the barbed bisimilarity on the $\chi$-processes. So we have in fact given a complete system for the barbed congruence. It is surprising that axiomatization of the barbed congruence is almost as difficult as axiomatization of all the 18 $L$-congruences. This points out the importance of the bisimulation lattice. Even if we do not care much about most of the $L$-congruences, we are forced to pay attention to them. The author certainly could not have discovered the axioms for 
barbed congruence had he not discovered the bisimulation lattice of $\chi$-calculus. As a digression, we remark that barbed bisimilarity, which we believe is a very sensible equality, is usually much weaker than the 'traditional bisimilarity' we have in mind. The coincidence, as might be the case in $\pi$-calculus with binary choice operator, is an exception rather than the rule.

The mistake made in $[11,3]$ is caused by the false assumption that Hennessy Lemma held in calculi of mobile processes. The result of this paper indicates that none of the systems given in $[11,3]$ is likely to be complete for the intended congruence. It is apparent from our work that Sangiorgi's system for strong open congruence on $\pi$-processes can be extended to a complete system for weak open congruence on $\pi$-processes by adding the tau laws and the promotion axiom Pr1.

Among the 17 prefix laws, P8, P9, P10 and P11 are most unusual. They share structural similarity that is quite different from the structures of the three tau laws shared by the rest of the laws. We leave for future study the question of if these laws can be simplified. The rest of the prefix laws are quite satisfactory.

The promotion axioms are very interesting. It is worth investigating the possibility of simplifying them.

Finally we remark that the prefix laws are not independent. For instance P6 is subsumed by $\mathrm{P} 7$ in the system $A S$. This however does not mean that $\mathrm{P} 6$ is redundant. It is used for example in the complete system of $={ }_{r o \cup i \cup f i \cup f o \cup u}$ for which $\mathrm{P} 7$ is too strong.

\section{References}

1. Fu, Y.: The $\chi$-Calculus. Proceedings of the International Conference on Advances in Parallel and Distributed Computing, March 19-21, Shanghai, IEEE Computer Society Press (1997) 74-81

2. Fu, Y.: A Proof Theoretical Approach to Communications. ICALP'97, July 7-11, Bologna, Italy, Lecture Notes in Computer Science 1256 (1997) 325-335

3. Fu, Y.: Bisimulation Lattice of Chi Processes. ASIAN'98, December 8-10, Manila, The Philippines, Lecture Notes in Computer Science 1538 (1998) 245-262

4. Fu, Y.: Reaction Graphs. Journal of Computer Science and Technology 13 (1998) $510-530$

5. Fu, Y.: Variations on Mobile Processes. To appear in Theoretical Computer Science, Elsevier Science Publisher

6. Fu, Y.: Algebraic Theory of Chi Calculus. Preprint

7. Lin, H.: Complete Inference Systems for Weak Bisimulation Equivalences in the $\pi$-Calculus. TAPSOFT'95, Lecture Notes in Computer Science 915 (1995) 187-201

8. Milner, R., Parrow, J., Walker, D.: A Calculus of Mobile Processes. Information and Computation 100 (1992) Part I:1-40, Part II:41-77

9. Milner, R., Sangiorgi, D.: Barbed Bisimulation. ICALP'92, Lecture Notes in Computer Science 623 (1992) 685-695

10. Parrow, J., Victor, B.: The Update Calculus. AMAST'97, December 13-17, Sydney, Australia, Lecture Notes in Computer Science 1119 (1997) 389-405

11. Parrow, J., Victor, B.: The Tau-Laws of Fusion. CONCUR'98, Lecture Notes in Computer Science 1466 (1998) 99-114

12. Sangiorgi, D.: A Theory of Bisimulation for $\pi$-Calculus. Acta Informatica 3 (1996) 69-97 